\title{
Fungal zinc metabolism and its connections to virulence
}

\author{
Charley C. Staats ${ }^{1,2 *}$, Lívia Kmetzsch ${ }^{1}$, Augusto Schrank ${ }^{1,2}$ and Marilene H. Vainstein ${ }^{1,2}$ \\ ${ }^{1}$ Centro de Biotecnologia, Universidade Federal do Rio Grande do Sul, Porto Alegre, Brazil \\ ${ }^{2}$ Departamento de Biologia Molecular e Biotecnologia, Universidade Federal do Rio Grande do Sul, Porto Alegre, Brazil
}

Edited by:

Frédéric J. Veyrier, Institut Pasteur,

France

Reviewed by:

Dario S. Zamboni, Universidade de

São Paulo, Brazil

Aaron Mitchell, Carnegie Mellon

University, USA

\section{${ }^{*}$ Correspondence:}

Charley C. Staats, Centro de

Biotecnologia, Universidade Federal

do Rio Grande do Sul, Av. Bento

Gonçalves 9500, 43421, Caixa

Postal 15005, Porto Alegre RS

91501-970, Brazil

e-mail:staats@cbiot.ufrgs.br
Zinc is a ubiquitous metal in all life forms, as it is a structural component of the almost $10 \%$ of eukaryotic proteins, which are called zinc-binding proteins. In zinc-limiting conditions such as those found during infection, pathogenic fungi activate the expression of several systems to enhance the uptake of zinc. These systems include ZIP transporters (solute carrier 39 family) and secreted zincophores, which are proteins that are able to chelate zinc. The expression of some fungal zinc uptake systems are regulated by a master regulator (Zap1), first characterized in the yeast Saccharomyces cerevisiae. In this review, we highlight features of zinc uptake and metabolism in human fungal pathogens and aspects of the relationship between proper zinc metabolism and the expression of virulence factors and adaptation to the host habitat.

Keywords: zinc ZIP transporters, zinc metabolism, zinc deprivation, ZAP transcription factor, fungal virulence

\section{INTRODUCTION}

Zinc is fundamental for all domains of life, as it composes the catalytic and structural center of a large array of proteins. Thus, the "zinc quota," defined as the cellular zinc content required for optimal growth (Outten and O'halloran, 2001), must be finely tuned. There is a huge variation in the number of zinc atoms per cell in different organisms $\left(10^{5}\right.$ for Escherichia coli, $10^{7}$ for yeast, and $10^{8}$ for mammalian cells). However, given cell size variation, zinc concentration is kept in close limits $(0.1-0.5 \mathrm{mM})$ (Eide, 2006). The zinc quota is maintained by the activity of specific membrane transporters or by zinc-binding proteins that mediate zinc uptake or storage. Zinc-depleting conditions are known to reduce fungal growth (Lulloff et al., 2004) and evidence suggests that host cells employ sequestration of zinc to hamper fungal development. This is exemplified by the reduced zinc levels in macrophages infected with Histoplasma capsulatum (Winters et al., 2010), and neutrophils infected with Cryptococcus neoformans enhance the production of calprotectin, a zinc-binding protein (Mambula et al., 2000). However, excess cellular zinc can generate an imbalance in oxidative metabolism (Pagani et al., 2007). Indeed, macrophages have developed a strategy to kill phagocytosed bacterial cells by zinc overload in the phagosomal environment and the consequent generation of high levels of reactive oxygen species (ROS) in the invading microorganisms (Botella et al., 2011). Here, we focused on the proteins involved in zinc metabolism in the fungal pathogens Aspergillus fumigatus, Candida albicans, C. neoformans, and C. gattii as compared to the well-characterized zinc metabolism-associated proteins from S. cerevisiae. Moreover, a critical appraisal on the participation of zinc metabolism and associated proteins in the establishment of fungal infections is presented.

\section{ZINC-ASSOCIATED BIOLOGICAL PROCESSES IN FUNGI}

There is little labile zinc inside cells; the estimated concentration of zinc in cells is in the picomolar to the nanomolar range
(Eide, 2006), and it is assumed that virtually all cellular zinc is associated with zinc-binding proteins. Bioinformatic analyzes to evaluate the presence of canonical sequences related to zinc binding have revealed that $5-6 \%$ of the predicted proteomes of prokaryotes consist of zinc-binding proteins, while this proportion reached 9\% in eukaryotic proteomes (Andreini et al., 2009). Using the fungal model S. cerevisiae, with a manually curated annotation of the genome and considerable biochemical information (Cherry et al., 2012), it is possible to infer the fraction of the proteome that can bind zinc. In fact, analyzes of predicted gene products from different organisms using the term "zinc ion binding" as a query of Gene Ontology databases revealed that some fungal species have a proportion of zinc-binding proteins that corresponds to $\sim 5 \%$ of the proteome (Figure 1). Considering only the zinc-binding proteins from S. cerevisiae, a large proportion of these proteins $(25 \%)$ are associated with biological processes related to transcriptional regulation. An even higher proportion of these proteins have the ability to bind DNA as accessed by Gene Ontology analysis (Figure 1). These numbers and the accumulation of a significant number of experimental reports point to the central role of zinc in gene expression regulation.

Consistent with the plethora of zinc-binding proteins associated with the regulation of gene expression in S. cerevisiae, a large number of these zinc-binding proteins consist of zinc finger transcription factors, the largest family of transcriptional regulators. Analysis of the Fungal Transcription Factor Database (Park et al., 2008) revealed that fungal species are characterized by a high diversity of zinc finger transcription factors, ranging from 116 in C. albicans to 311 in A. fumigatus (Figure 1). The vast majority of these transcription factors belong to the widely phylogenetically distributed Zn2Cys6. In S. cerevisiae, members of this family are involved in the regulation of several biological processes, including sugar and amino acid metabolism, nitrogen utilization, mitosis, and meiosis (Macpherson et al., 2006). However, 


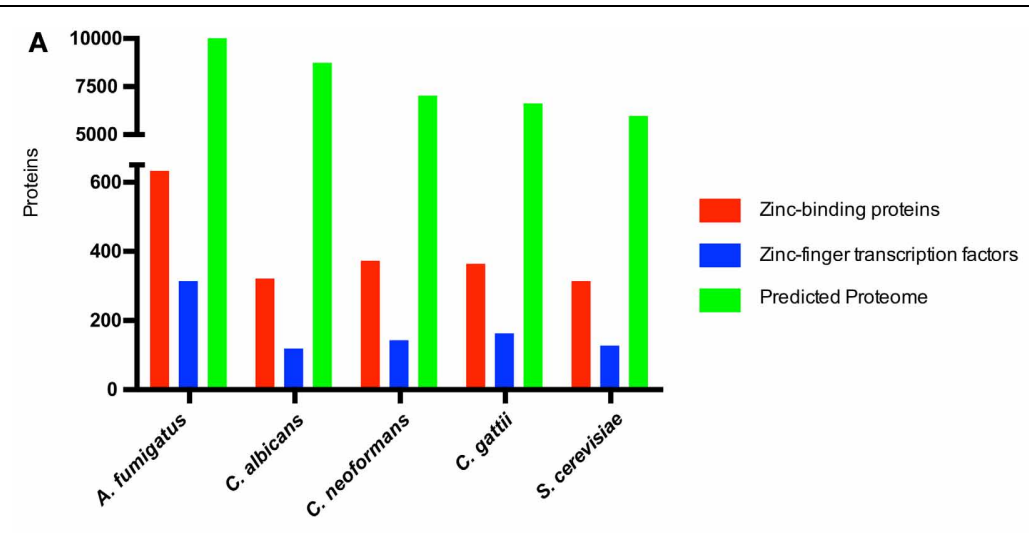

B

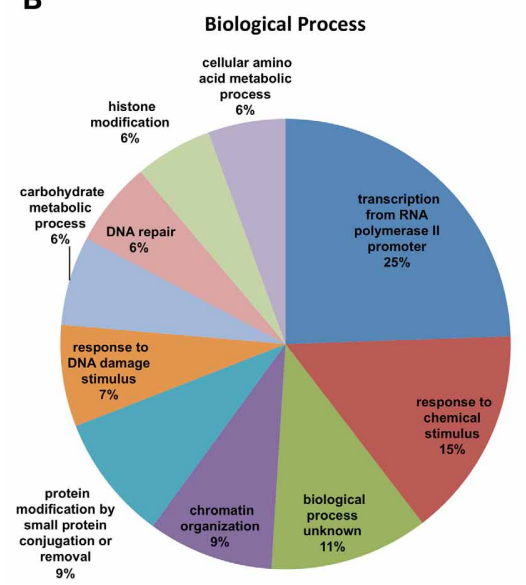

FIGURE 1 | Zinc-binding proteins associated biological processes in

fungi. (A) The number of zinc finger transcription factors and zinc-binding proteins compared to the total proteome from fungi. The predicted proteomes of $A$. fumigatus, $C$. albicans, $C$. neoformans, $C$. gattii, and $S$. cerevisiae were retrieved from their respective genome assemblies in NCBI (Accession numbers GCA_000002655.1, GCA_000182965.2, GCA_000149245.2, GCA_000185945.1, GCA_000146045.2, respectively). The protein sequences

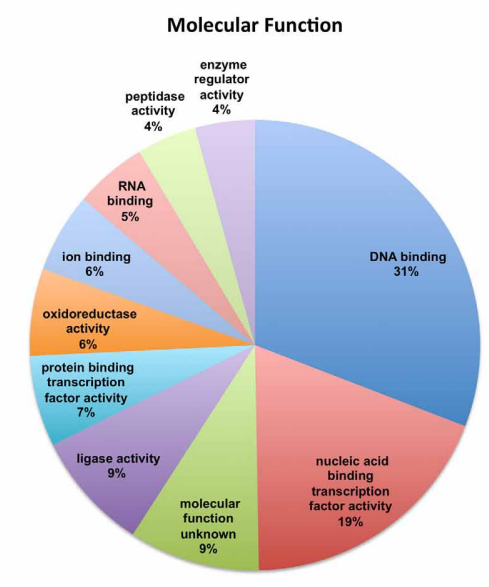

were submitted to the UFO webserver (Meinicke, 2009) for functional profiling. All proteins with the Gene Ontology annotation "zinc ion binding" (GO:0008270) were considered to be zinc-binding proteins. (B) The 311 yeast proteins annotated as zinc-binding proteins in the Yeast Genome Database were submitted to GO-slim analysis (Cherry et al., 2012). The 10 most abundant biological process (left graph) and molecular function (right graph) associations were considered, and the fraction of proteins in each class is depicted. other biological processes are associated with zinc-binding proteins. This is consistent with the enzymatic activity present in some zinc-binding proteins, including ligase, peptidase, and oxidoreductase activities (Figure 1). Yet in S. cerevisiae, the $\mathrm{Cu} / \mathrm{Zn}$ superoxide dismutase and the alcohol dehydrogenase (Adh1) proteins are the most abundant zinc-binding proteins (Eide, 2006).

Some zinc-binding proteins are also involved in fungal virulence. Superoxide dismutases (Sods) are the central enzymes in fungi associated with the detoxification of ROS generated by host cells during host-pathogen interactions (Huang et al., 2009). In this view, specific Sods from pathogenic fungi are assumed to be virulence determinants. C. albicans expresses six superoxide dismutase isoforms (Sod1-Sod6), four of which are annotated as copper/zinc-dependent enzymes (Sod1, Sod4, Sod5, and Sod6) (Frohner et al., 2009). Functional analysis has revealed that Sod1, Sod4, and Sod5 are necessary for the proper detoxification of ROS by C. albicans, as SOD-null mutants displayed growth defects in the presence of ROS-generating compounds. In addition, these mutants displayed increased susceptibility to macrophage killing and reduced virulence (Hwang et al., 2002; Martchenko et al., 2004; Frohner et al., 2009). The A. fumigatus genome contains four genes encoding Sods, two of which are annotated as copper/zinc-dependent (Sod1 and Sod4). A. fumigatus cells lacking the SOD1 gene are hypersensitive to menadione, a ROS generating agent, but the virulence of cells lacking this gene is not affected (Lambou et al., 2010). In C. neoformans, two Sodencoding genes have been described. The SOD1 gene encodes a copper/zinc-dependent Sod required for full virulence in animal models of cryptococcosis and for survival inside macrophages (Cox et al., 2003).

Zinc-binding metalloproteases have also shown to be involved in virulence. Distinct species of pathogenic fungi secrete proteases during the infection. These proteases are classified into aspartic proteases, serine proteases, and metalloproteases (Yike, 2011). The deuterolysin (M35) family of metalloproteases is characterized by the presence of two zinc-binding histidines and a catalytic glutamate in their catalytic centers (Markaryan et al., 1994). The roles of metalloproteases secreted by pathogenic fungi 
are largely associated with tissue degradation. This is evident for the Mep3 metalloprotease from Microsporum canis (Brouta et al., 2001). The ADAM proteases (from A Disintegrin And Metalloproteinase) belongs to the M12 family of metalloproteases according to the MEROPS database (Rawlings et al., 2012). These proteins are produced as pro-enzymes that must be secreted and activated prior to performing their biological functions. ADAM proteases have been implicated in several aspects of cell biology including adhesion, migration, proteolysis, and signaling (Edwards et al., 2008). The presence of two copies of putative ADAM coding sequences in the genome of $A$. fumigatus indicates a possible contribution for this family in virulence in this fungus. However, no functional characterization was performed yet to evaluate whether ADAM proteases can be associated to virulence in A. fumigatus.

\section{FUNGAL ZINC UPTAKE}

Fungal cells must acquire zinc for proper development during their life cycle, even when they are saprophytes or during the infection process. To hamper pathogen growth, mammalian hosts typically reduce the levels of free zinc and other metals (Kehl-Fie and Skaar, 2010). The concentration of zinc in human tissues varies dramatically, ranging from $10 \mu \mathrm{g} / \mathrm{g}$ (lungs) to $83.2 \mu \mathrm{g} / \mathrm{g}$ (liver). In body fluids, the zinc concentration ranges from 0.2 to $8.7 \mu \mathrm{g} / \mathrm{mL}$ (Lech and Sadlik, 2011). Thus, pathogenic fungi have developed efficient strategies to uptake zinc to overcome the limits imposed by host.

The initial characterization of zinc transport mechanisms in fungi was done in $S$. cerevisiae and revealed the central role of the ZIP (Zrt-, Irt-like protein) family of zinc transporters (Eide, 2006). The name of this family, also known as SLC39 (solute carrier 39), refers to the first members to be functionally characterized, the S. cerevisiae zinc transporters Zrt1 and Zrt2 and the Arabidopsis thaliana iron transporter Irtl (Eide, 2004). ZIP family transporters are associated with zinc transport into the cytoplasm across cellular membranes, either from the extracellular space or from within organelles. ZIP transporters are characterized by eight putative transmembrane regions, and the amino- and carboxyl-termini are often located on the extracellular or luminal side of membranes (Eide, 2004). A histidine-rich region present between transmembrane regions three and four is necessary for zinc selectivity, as demonstrated for the TjZNT1 ZIP transporter from the nickel hyperaccumulator plant Thlaspi japonicum (Nishida et al., 2008).

The S. cerevisiae Zrt1 is a high-affinity zinc transporter that is expressed when cells are cultivated in low-zinc media (Zhao and Eide, 1996a), while the low-affinity transporter Zrt2 mediates the uptake of zinc, cooper, and iron (Zhao and Eide, 1996b). Additional non-specific zinc transporters are also associated with zinc uptake, as zrt1/zrt2 double-mutants are capable of growing in low-zinc conditions (Zhao and Eide, 1996b). These transporters include the low-affinity iron transporter Fet 4 and the phosphate transporter Pho84, which mediate the uptake of zinc by phosphate chelation of this metal (Waters and Eide, 2002; Jensen et al., 2003). Inside cells, zinc is shuttled to different compartments, including the nucleus, endoplasmic reticulum and vacuole, by the activity of specific transporters not related to the ZIP family
(Eide, 2009). Zrt3 is a ZIP transporter found in the membranes of vacuoles that accumulate zinc, the so-called "zincosomes." The function of Zrt3 is associated supplying zinc to the cytoplasm from zincosomes (Simm et al., 2007).

The number of ZIP genes in the genomes of A. fumigatus, C. albicans, C. neoformans, and C. gattii ranges from four to nine. Genome annotation based evidences suggest that many of these transporters show high similarity to S. cerevisiae Zrt1 or Zrt2 proteins, suggesting that both low- and high-affinity zinc uptake systems exist in these pathogenic fungi. In A. fumigatus, three ZIP zinc transporters have been characterized. The expression of $z r f A$ and $z r f B$ genes is activated by low levels of zinc or iron. In addition, the expression of these transporters in response to zinc deprivation occurs mainly in an acidic environment. Null mutants for these genes, as well as double mutants lacking both genes, showed a reduced ability to grow under zinc deprivation (Vicentefranqueira et al., 2005). Further analysis showed that the expression of these genes is under control of the $\mathrm{pH}$ homeostasis regulator PacC (Amich et al., 2009). A third zinc transporter from A. fumigatus, which is encoded by the gene $z r f C$, is expressed during zinc-deprivation conditions when the fungus is grown in alkaline $\mathrm{pH}$ conditions. Null mutants of this gene are severely reduced in their ability to grow during zinc deprivation (Amich et al., 2010). In C. gattii, zinc deprivation by the chelator N,N,N,N-tetrakis(2-pyridylmethyl)ethylenediamine (TPEN) induced the expression of the putative zinc transporters encoded by genes ZIP1, ZIP2, and ZIP3 (Schneider et al., 2012).

Some fungal species possess additional mechanisms to sequester zinc from host cells and tissues in a process analogous to iron chelation by secreted siderophores. C. albicans secretes the antigenic protein Pra1, a zinc-binding protein that is able to scavenge zinc from tissues invaded by the fungus. Moreover, molecular docking experiments revealed that Pral could interact with the zinc transporter Zrt1. Pral participates in proper endothelial colonization by C. albicans (Citiulo et al., 2012) and is associated with evasion of immune cells (Luo et al., 2011; Soloviev et al., 2011). In addition, orthologs of the gene encoding Pral are found in diverse fungal pathogens (Citiulo et al., 2012) including A. fumigatus (the zinc-regulated aspf2 gene (Amich et al., 2010). However, no orthologs could be found in C. neoformans or C. gattii. These zinc-sequestering genes are generally clustered with Zrt1 orthologs in these fungi in a highly syntenic fashion (Citiulo et al., 2012), representing a conserved mechanism for zinc acquisition during host-fungal interactions.

\section{EFFECTS OF ZINC DEPRIVATION ON FUNGAL CELLS}

Zinc chelation is able to reduce fungal growth in both rich and defined media (Lulloff et al., 2004). In fact, zinc chelation is assumed to occur during infection and is an important strategy developed by immune cells to hamper pathogen growth (Corbin et al., 2008). Zinc restriction by host cells is achieved by lowering metal availability via the activity of the host zinc transporters or the expression of zinc-binding proteins. An example of a zincbinding protein that is expressed to reduce the bioavailability of zinc is calprotectin, a member of the S100 family of metalbinding proteins (Goyette and Geczy, 2011). Calprotectin was found to reduce the growth of diverse fungal species in vitro 
(Lulloff et al., 2004). Moreover, this protein is produced by neutrophils in order to reduce the development of $A$. fumigatus (McCormick et al., 2010), C. albicans (Urban et al., 2009), and C. neoformans (Mambula et al., 2000). Neutrophils are able to kill invading pathogens by phagocytosis, secreting anti-microbial molecules and forming neutrophil extracellular traps (NETs). NET formation is derived from a distinct mechanism of cell death that is characterized by the loss of intracellular membranes and further structural derange of the plasma membrane. As a result of this loss of membrane functionality, NETs are composed of nucleosomes and a set of cytoplasmic and granular interacting proteins (Brinkmann and Zychlinsky, 2012). NETs formed in response to A. fumigatus and C. albicans infection contain calprotectin, and the presence of this protein in such structures is fundamental for the proper antifungal activity of neutrophils (Urban et al., 2009; McCormick et al., 2010).

The direct effects of zinc deprivation on fungal cells are poorly understood. Assays employing S. cerevisiae revealed that, by an unknown mechanism, cells that are exposed to zinc deprivation experience increased levels of ROS (Wu et al., 2007). However, yeast cells employ different strategies to cope with the stress caused by zinc deprivation. As revealed by transcriptomic and functional analyzes in $S$. cerevisiae, low zinc conditions lead to alterations in lipid synthesis, methionine, and sulfate metabolism and to oxidative stress tolerance (Iwanyshyn et al., 2004; Wu et al., 2007, 2009). Furthermore, a genome-wide functional analysis employing a $S$. cerevisiae mutant library that encompasses more than 4500 gene knockout mutants revealed that almost 400 different gene products are necessary for proper growth in zinc-limiting conditions. Among these gene products are those associated with the oxidative stress response, endoplasmic reticulum function, peroxisome biogenesis, histone deacetylation, and zinc uptake (North et al., 2012). Zinc deprivation induced by TPEN also induced accumulation of intracellular ROS in C. gattii cells (Schneider et al., 2012). Moreover, proteomic profiling of the dimorphic fungus Paracoccidioides brasiliensis exposed to TPEN also revealed an increased expression of proteins involved in stress tolerance, suggesting that zinc deprivation induces stress in these cells (Parente et al., 2013). Thus, it is reasonable to suggest that zinc deprivation hampers fungal development by restricting the activity of zinc-binding proteins and by submitting the fungal cells to different kinds of stress.

\section{REGULATION OF ZINC HOMEOSTASIS}

The transcriptional responses to zinc deprivation of fungal cells are regulated by the Zap 1 transcription factor. The first characterization of the role of Zap1 in regulating zinc homeostasis was performed in S. cerevisiae (Zhao and Eide, 1997). This major zinc metabolism regulator contains seven $\mathrm{C}_{2} \mathrm{H}_{2}$ zinc finger domains (ZF). While the domains ZF3-ZF7, located to C-terminal region, are directly associated with the recognition and binding of zincresponsive elements (ZRE) in the promoters of Zap1-regulated genes, $\mathrm{ZF} 1$ and $\mathrm{ZF} 2$ lie in the zinc-responsive element present in the activation domain (AD) of this transcription factor. Zinc binding to $\mathrm{ZFs}$ in $\mathrm{AD} 2$ of Zap1 represses the activity of this transcription factor and therefore inhibits the expression of Zap1regulated genes (Bird et al., 2003; Herbig et al., 2005). However, zinc can also influence the binding of Zap1 to its ZREs by modulating of the activity of ZF3-ZF7 (Frey et al., 2011). Zap1 activates the expression of more than 60 genes in response to low-zinc environment, including genes that code for zinc transporters $(Z R T 1$, ZRT2, ZRT3, ZRC1, and FET4), proteins related to stress (CTT1, $T S A 1$, and HSP26), as well as regulating its own expression (Lyons et al., 2000; Wu et al., 2008).

Orthologs of S. cerevisiae Zap1 were functionally characterized in three pathogenic fungal species. The A. fumigatus zafA expression is induced in zinc-limiting media and repressed by zinc. In addition, null mutants of $z a f A$ have a reduced ability to grown in low-zinc media as a direct effect of diminished zinc transport. Consequently, such mutants displayed a complete lack of virulence in murine models of aspergillosis. In addition, no conidial germination could be observed in mice infected with mutant cells lacking zafA (Moreno et al., 2007).

The C. albicans Csr1/Zap1 transcription factor was also shown to influence growth in zinc-deprivation conditions and affect important virulence traits in this pathogenic yeast. While cells lacking the CSR1/ZAP1 gene displayed reduced filamentation, the same mutants showed increased $\beta$-glucan content in their biofilm matrices. Moreover, the biofilms produced by such cells had a predominance cells in the yeast form when compared to wild-type biofilms (Kim et al., 2008; Nobile et al., 2009). The characterization of Csr1/Zap1 targets in biofilm-inducing conditions by chromatin immunoprecipitation analysis showed that this transcription factor recognizes the promoters of approximately 60 genes including the ZRT1, ZRT2, and ZRT3 genes (zinc transporters) and the CSR1/ZAP1 gene itself (Nobile et al., 2009). Further characterization of Csr1/Zap1 revealed that this transcription factor also regulates cell-cell signaling during biofilm development by regulating the expression of Dpp1, a farnesol synthesis protein. Farnesol is an important mediator of biofilm formation as it acts as a quorum-sensing molecule that functions as an inhibitor of the yeast-to-hyphae transition in biofilms (Ganguly et al., 2011).

The Zap1 ortholog from C. gattii also regulates the expression of zinc uptake systems. Null mutants of this gene were defective in growth in zinc-deprivation conditions, accumulated intracellular ROS, were hypersensitive to reactive nitrogen species, and displayed attenuated virulence in murine inhalation models of cryptococcosis. Transcriptomic profiling of wild type and zap1-null mutants exposed to TPEN revealed that more than 500 genes were differentially expressed between these cell types. The large majority of these differentially expressed gene products were found to be related to adaptive responses to zinc deprivation, but inferred true Zap1 targets were also found including the zinc transporters encoded by ZIP1 and ZIP2 (Schneider et al., 2012).

A direct comparison between of C. albicans, C. gattii, and S. cerevisiae Zap1-dependent transcription profiles revealed a common Zap1 regulon (Table 1). Among positively regulated genes, figure out the genes for the zinc acquisition systems, while a large proportion of negatively regulated genes consist of zinc-binding proteins (Wu et al., 2008; Nobile et al., 2009; Schneider et al., 2012). These proteins include a large family of alcohol dehydrogenases, which are most likely the most 
Table 1 | Common set of genes regulated by Zap1 orthologs in yeasts.

\begin{tabular}{|c|c|c|c|c|c|c|}
\hline Functional category & \multicolumn{2}{|c|}{ S. cerevisiae } & \multicolumn{2}{|c|}{ C. albicans } & \multicolumn{2}{|c|}{ C. gattii } \\
\hline Zinc transport & ZRT2 & 3.43 & ZRT2 & 4.75 & ZIP2 (CNAG_2209) & 6.96 \\
\hline Zinc conservation & XYL2 & -1.32 & BZD99 & -0.96 & CNBG_3878 & -2.73 \\
\hline
\end{tabular}

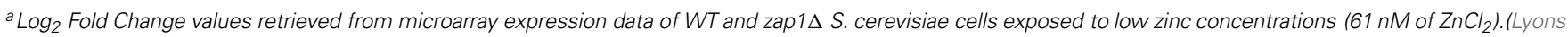
et al., 2000).

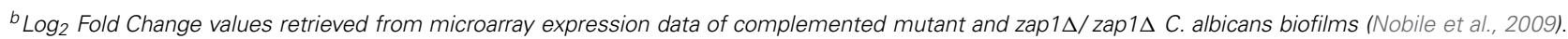

${ }^{c} \mathrm{Log}_{2}$ Fold Change values retrieved from RNA-Seq expression data of WT and zap1 $\mathrm{C}$. gattii cells exposed to a zinc chelator (10 $\mu$ M of TPEN).(Schneider et al., 2012).

abundant zinc-binding proteins in the cell. This trend represents a conserved fungal strategy to shuttle zinc into essential proteins specialized for zinc conservation. This mechanism is characterized by the down-regulation of the expression of abundant zinc storage proteins, such as zinc-dependent alcohol dehydrogenases, in zinc-deprivation conditions. In this way, zinc can easily be mobilized to other zinc-dependent proteins necessary for proper development under these harsh conditions (Eide, 2009).

\section{CONCLUSIONS AND FUTURE STUDIES}

Zinc influences diverse mechanisms of fungal pathogenesis by directly associating with virulence determinants (i.e., metalloproteases or Sods) or by regulating the expression of many

\section{REFERENCES}

Amich, J., Leal, F., and Calera, J. A. (2009). Repression of the acid ZrfA/ZrfB zinc-uptake system of Aspergillus fumigatus mediated by PacC under neutral, zinc-limiting conditions. Int. Microbiol. 12, 39-47.

Amich, J., Vicentefranqueira, R., Leal, F., and Calera, J. A. (2010). Aspergillus fumigatus survival in alkaline and extreme zinc-limiting environments relies on the induction of a zinc homeostasis system encoded by the $\mathrm{zrfC}$ and aspf2 genes. Eukaryotic Cell 9, 424-437. doi: 10.1128/EC.00348-09

Andreini, C., Bertini, I., and Rosato, A. (2009). Metalloproteomes: a bioinformatic approach. Acc. Chem. Res. 42, 1471-1479. doi: 10.1021/ar900015x

Bird, A. J., McCall, K., Kramer, M., Blankman, E., Winge, D. R., and Eide, D. J. (2003). Zinc fingers can act as $\mathrm{Zn} 2+$ sensors to regulate transcriptional activation domain function. EMBO J. 22, 5137-5146. doi: $10.1093 / \mathrm{emboj} / \mathrm{cdg} 484$

Botella, H., Peyron, P., Levillain, F., Poincloux, R., Poquet, Y., Brandli,
I., et al. (2011). Mycobacterial $\mathrm{p}$ (1)-type ATPases mediate resistance to zinc poisoning in human macrophages. Cell Host Microbe 10, 248-259. doi: 10.1016/j.chom.2011.08.006

Brinkmann, V., and Zychlinsky, A. (2012). Neutrophil extracellular traps: is immunity the second function of chromatin? J. Cell Biol. 198, 773-783. doi: 10.1083/jcb.201203170

Brouta, F., Descamps, F., Fett, T., Losson, B., Gerday, C., and Mignon, B. (2001). Purification and characterization of a $43.5 \mathrm{kDa}$ keratinolytic metalloprotease from Microsporum canis. Med. Mycol. 39, 269-275. doi: 10.1080/714031031

Cherry, J. M., Hong, E. L., Amundsen, C., Balakrishnan, R., Binkley, G., Chan, E. T., et al. (2012). Saccharomyces genome database: the genomics resource of budding yeast. Nucleic Acids Res. 40, D700-D705. doi: 10.1093/nar/gkr1029

Citiulo, F., Jacobsen, I. D., Miramon, P., Schild, L., Brunke, S., Zipfel, P., et al. (2012). Candida albicans scavenges host zinc via Pral

proteins required for infection. The regulation of zinc acquisition by the Zap1 transcription factors is fundamental for fungal pathogenesis in mammalian hosts. A broader functional characterization of Zip transporters in fungi, including plant and insect fungal pathogens, will elucidate the pivotal role of pathogen zincbinding proteins during the infectious process. As active zinc deprivation by hosts represents an important antifungal mechanism, development of chelating strategies to control in vivo fungal development may be a plausible chemotherapeutic alternative.

\section{ACKNOWLEDGMENTS}

This work was supported by grants from the Brazilian agencies CNPq, FINEP, and CAPES.

during endothelial invasion. PLoS Pathog. 8:e1002777. doi: 10.1371/journal.ppat.1002777

Corbin, B. D., Seeley, E. H., Raab, A., Feldmann, J., Miller, M. R., Torres, V. J., et al. (2008). Metal chelation and inhibition of bacterial growth in tissue abscesses. Science 319, 962-965. doi: 10.1126/science.1152449

Cox, G. M., Harrison, T. S., McDade, H. C., Taborda, C. P., Heinrich, G., Casadevall, A., et al. (2003). Superoxide dismutase influences the virulence of Cryptococcus neoformans by affecting growth within macrophages. Infect. Immun. 71, 173-180. doi: 10.1128/IAI.71.1.173180.2003

Edwards, D. R., Handsley, M. M., and Pennington, C. J. (2008). The ADAM metalloproteinases. Mol. Aspects Med. 29, 258-289. doi: 10.1016/j.mam.2008.08.001

Eide, D. J. (2004). The SLC39 family of metal ion transporters. Pflugers Arch. 447, 796-800. doi: 10.1007/s00424-003-1074-3

Eide, D. J. (2006). Zinc transporters and the cellular trafficking of zinc. Biochim. Biophys.
Acta 1763, 711-722. doi: 10.1016/j.bbamcr.2006.03.005

Eide, D. J. (2009). Homeostatic and adaptive responses to zinc deficiency in Saccharomyces cerevisiae. J. Biol. Chem. 284, 18565-18569. doi: 10.1074/jbc. R900014200

Frey, A. G., Bird, A. J., Evans-Galea, M. V., Blankman, E., Winge, D. R., and Eide, D. J. (2011). Zincregulated DNA binding of the yeast Zap1 zinc-responsive activator. PLoS ONE 6:e22535. doi: 10.1371/journal.pone.0022535

Frohner, I. E., Bourgeois, C., Yatsyk, K., Majer, O., and Kuchler, K. (2009). Candida albicans cell surface superoxide dismutases degrade host-derived reactive oxygen species to escape innate immune surveillance. Mol. Microbiol. 71, 240-252. doi: 10.1111/j.1365-2958.2008.06528.x

Ganguly, S., Bishop, A. C., Xu, W., Ghosh, S., Nickerson, K. W., Lanni, F., et al. (2011). Zap1 control of cell-cell signaling in Candida albicans biofilms. Eukaryotic Cell 10, 1448-1454. doi: 10.1128/EC.05196-11 
Goyette, J., and Geczy, C. L. (2011). Inflammation-associated S100 proteins: new mechanisms that regulate function. Amino Acids 41, 821-842. doi: 10.1007/s00726-010-0528-0

Herbig, A., Bird, A. J., Swierczek, S., McCall, K., Mooney, M., Wu, C. Y., et al. (2005). Zap1 activation domain 1 and its role in controlling gene expression in response to cellular zinc status. Mol. Microbiol. 57, 834-846. doi: 10.1111/j.13652958.2005.04734.x

Huang, J., Canadien, V., Lam, G. Y., Steinberg, B. E., Dinauer, M. C., Magalhaes, M. A., et al. (2009). Activation of antibacterial autophagy by NADPH oxidases. Proc. Natl. Acad. Sci. U.S.A. 106, 6226-6231. doi: 10.1073/pnas.0811045106

Hwang, C. S., Rhie, G. E., Oh, J. H., Huh, W. K., Yim, H. S., and Kang, S. O. (2002). Copperand zinc-containing superoxide dismutase $(\mathrm{Cu} / \mathrm{ZnSOD})$ is required for the protection of Candida albicans against oxidative stresses and the expression of its full virulence. Microbiology 148, 3705-3713.

Iwanyshyn, W. M., Han, G. S., and Carman, G. M. (2004). Regulation of phospholipid synthesis in Saccharomyces cerevisiae by zinc. J. Biol. Chem. 279, 21976-21983. doi: 10.1074/jbc.M402047200

Jensen, L. T., Ajua-Alemanji, M., and Culotta, V. C. (2003). The Saccharomyces cerevisiae high affinity phosphate transporter encoded by PHO84 also functions in manganese homeostasis. J. Biol. Chem. 278, 42036-42040. doi: 10.1074/jbc.M307413200

Kehl-Fie, T. E., and Skaar, E. P. (2010). Nutritional immunity beyond iron: a role for manganese and zinc. Curr. Opin. Chem. Biol. 14, 218-224. doi: 10.1016/j.cbpa.2009.11.008

Kim, M. J., Kil, M., Jung, J. H., and Kim, J. (2008). Roles of Zincresponsive transcription factor Csrl in filamentous growth of the pathogenic Yeast Candida albicans. J. Microbiol. Biotechnol. 18, 242-247.

Lambou, K., Lamarre, C., Beau, R., Dufour, N., and Latge, J. P. (2010). Functional analysis of the superoxide dismutase family in Aspergillus fumigatus. Mol. Microbiol. 75, 910-923. doi: 10.1111/j.1365-2958.2009.07024.x

Lech, T., and Sadlik, J. K. (2011). Zinc in postmortem body tissues and fluids. Biol. Trace Elem. Res. 142, 11-17. doi: 10.1007/s12011010-8747-5
Lulloff, S. J., Hahn, B. L., and Sohnle, P. G. (2004). Fungal susceptibility to zinc deprivation. J. Lab. Clin. Med. 144, 208-214. doi: 10.1016/j.lab.2004.07.007

Luo, S., Blom, A. M., Rupp, S., Hipler, U. C., Hube, B., Skerka, C., et al. (2011). The pH-regulated antigen 1 of Candida albicans binds the human complement inhibitor C4b-binding protein and mediates fungal complement evasion. J. Biol. Chem. 286, 8021-8029. doi: 10.1074/jbc.M110. 130138

Lyons, T. J., Gasch, A. P., Gaither, L. A., Botstein, D., Brown, P. O., and Eide, D. J. (2000). Genomewide characterization of the Zaplp zinc-responsive regulon in yeast. Proc. Natl. Acad. Sci. U.S.A. 97, 7957-7962. doi: 10.1073/pnas.97.14.7957

Macpherson, S., Larochelle, M., and Turcotte, B. (2006). A fungal family of transcriptional regulators: the zinc cluster proteins. Microbiol. Mol. Biol. Rev. 70, 583-604. doi: 10.1128/MMBR. 00015-06

Mambula, S. S., Simons, E. R., Hastey, R., Selsted, M. E., and Levitz, S. M. (2000). Human neutrophil-mediated nonoxidative antifungal activity against Cryptococcus neoformans. Infect. Immun. 68, 6257-6264. doi: 10.1128/IAI.68.11.6257-6264.2000

Markaryan, A., Morozova, I., Yu, H., and Kolattukudy, P. E. (1994). Purification and characterization of an elastinolytic metalloprotease from Aspergillus fumigatus and immunoelectron microscopic evidence of secretion of this enzyme by the fungus invading the murine lung. Infect. Immun. 62, 2149-2157.

Martchenko, M., Alarco, A. M., Harcus, D., and Whiteway, M. (2004). Superoxide dismutases in Candida albicans: transcriptional regulation and functional characterization of the hyphal-induced SOD5 gene. Mol. Biol. Cell 15, 456-467. doi: 10.1091/mbc.E03-03-0179

McCormick, A., Heesemann, L., Wagener, J., Marcos, V., Hartl, D., Loeffler, J., et al. (2010). NETs formed by human neutrophils inhibit growth of the pathogenic mold Aspergillus fumigatus. Microbes Infect. 12, 928-936. doi: 10.1016/j.micinf.2010.06.009

Meinicke, P. (2009). UFO: a web server for ultra-fast functional profiling of whole genome protein sequences. BMC Genomics 10:409. doi: 10.1186/1471-2164-10-409
Moreno, M. A., Ibrahim-Granet, O., Vicentefranqueira, R., Amich, J., Ave, P., Leal, F., et al. (2007) The regulation of zinc homeostasis by the ZafA transcriptional activator is essential for Aspergillus fumigatus virulence. Mol. Microbiol. 64, 1182-1197. doi: 10.1111/j.13652958.2007.05726.x

Nishida, S., Mizuno, T., and Obata, H. (2008). Involvement of histidine-rich domain of ZIP family transporter TjZNT1 in metal ion specificity. Plant Physiol. Biochem. 46, 601-606. doi: 10.1016/j.plaphy.2008.02.011

Nobile, C. J., Nett, J. E., Hernday, A. D., Homann, O. R., Deneault, J. S., Nantel, A., et al. (2009). Biofilm matrix regulation by Candida albicans Zap1. PLoS Biol. 7:e1000133. doi: 10.1371/journal. pbio. 1000133

North, M., Steffen, J., Loguinov, A. V., Zimmerman, G. R., Vulpe, C. D., and Eide, D. J. (2012). Genome-wide functional profiling identifies genes and processes important for zinc-limited growth of Saccharomyces cerevisiae. PLoS Genet. 8:e1002699. doi: 10.1371/journal.pgen.1002699

Outten, C. E., and O'halloran, T. V. (2001). Femtomolar sensitivity of metalloregulatory proteins controlling zinc homeostasis. Science 292, 2488-2492. doi: 10.1126/science. 1060331

Pagani, M. A., Casamayor, A., Serrano, R., Atrian, S., and Arino, J. (2007). Disruption of iron homeostasis in Saccharomyces cerevisiae by high zinc levels: a genomewide study. Mol. Microbiol. 65, 521-537. doi: 10.1111/j.1365-2958. 2007.05807.x

Parente, A. F., De Rezende, T. C., De Castro, K. P., Bailao, A. M., Parente, J. A., Borges, C. L., et al. (2013). A proteomic view of the response of Paracoccidioides yeast cells to zinc deprivation. Fungal Biol 117, 399-410. doi: 10.1016/j.funbio.2013.04.004

Park, J., Park, J., Jang, S., Kim, S., Kong, S., Choi, J., et al. (2008). FTFD: an informatics pipeline supporting phylogenomic analysis of fungal transcription factors. Bioinformatics 24, 1024-1025. doi: 10.1093/bioinformatics/btn058

Rawlings, N. D., Barrett, A. J., and Bateman, A. (2012). MEROPS: the database of proteolytic enzymes, their substrates and inhibitors. Nucleic Acids Res. 40, D343-D350. doi: 10.1093/nar/gkr987

Schneider, R. O., Fogaca, N. S. S., Kmetzsch, L., Schrank, A.,
Vainstein, M. H., and Staats, C. C. (2012). Zapl regulates zinc homeostasis and modulates virulence in Cryptococcus gattii. PLoS ONE 7:e43773. doi: 10.1371/journal.pone.0043773

Simm, C., Lahner, B., Salt, D., Lefurgey, A., Ingram, P., Yandell, B., et al. (2007). Saccharomyces cerevisiae vacuole in zinc storage and intracellular zinc distribution. Eukaryot Cell 6, 1166-1177. doi: 10.1128/EC.00077-07

Soloviev, D. A., Jawhara, S., and Fonzi, W. A. (2011). Regulation of innate immune response to Candida albicans infections by alphaMbeta2-Pra1p interaction. Infect. Immun. 79, 1546-1558. doi: 10.1128/IAI.00650-10

Urban, C. F., Ermert, D., Schmid, M., Abu-Abed, U., Goosmann, C., Nacken, W., et al. (2009). Neutrophil extracellular traps contain calprotectin, a cytosolic protein complex involved in host defense against Candida albicans. PLoS Pathog. 5:e1000639. doi: 10.1371/journal.ppat.1000639

Vicentefranqueira, R., Moreno, M. A., Leal, F., and Calera, J. A. (2005). The zrfA and zrfB genes of Aspergillus fumigatus encode the zinc transporter proteins of a zinc uptake system induced in an acid, zinc-depleted environment. Eukaryotic Cell 4, 837-848. doi: 10.1128/EC.4.5.837848.2005

Waters, B. M., and Eide, D. J. (2002). Combinatorial control of yeast FET4 gene expression by iron, zinc, and oxygen. J. Biol. Chem. 277, 33749-33757. doi: 10.1074/jbc.M206214200

Winters, M. S., Chan, Q., Caruso, J. A., and Deepe, G. S. Jr. (2010). Metallomic analysis of macrophages infected with Histoplasma capsulatum reveals a fundamental role for zinc in host defenses. J. Infect. Dis. 202, 1136-1145. doi: 10.1086/656191

Wu, C. Y., Bird, A. J., Chung, L. M., Newton, M. A., Winge, D. R., and Eide, D. J. (2008). Differential control of Zap1regulated genes in response to zinc deficiency in Saccharomyces cerevisiae. BMC Genomics 9:370. doi: 10.1186/1471-21649-370

Wu, C. Y., Bird, A. J., Winge, D. R., and Eide, D. J. (2007). Regulation of the yeast TSAl peroxiredoxin by ZAP1 is an adaptive response to the oxidative stress of zinc deficiency. J. Biol. Chem. 282, 2184-2195. doi: 10.1074/jbc.M606639200 
Wu, C. Y., Roje, S., Sandoval, F. J., Bird, A. J., Winge, D. R., and Eide, D. J. (2009). Repression of sulfate assimilation is an adaptive response of yeast to the oxidative stress of zinc deficiency. J. Biol. Chem. 284, 27544-27556. doi: 10.1074/jbc.M109.042036

Yike, I. (2011). Fungal proteases and their pathophysiological effects. Mycopathologia 171, 299-323. doi: 10.1007/s11046-010-9386-2

Zhao, H., and Eide, D. (1996a). The yeast ZRT1 gene encodes the zinc transporter protein of a high-affinity uptake system induced by zinc limitation. Proc. Natl. Acad. Sci. U.S.A. 93, 2454-2458. doi: 10.1073/pnas.93. 6.2454

Zhao, H., and Eide, D. (1996b). The ZRT2 gene encodes the low affinity zinc transporter in Saccharomyces cerevisiae. J. Biol. Chem. 271, 23203-23210. doi: 10.1074/jbc.271.38.23203

Zhao, H., and Eide, D. J. (1997). Zaplp, a metalloregulatory protein involved in zinc-responsive transcriptional regulation in Saccharomyces cerevisiae. Mol. Cell. Biol. 17, 5044-5052.
Conflict of Interest Statement: The authors declare that the research was conducted in the absence of any commercial or financial relationships that could be construed as a potential conflict of interest.

Received: 26 July 2013; accepted: 26 September 2013; published online: 14 October 2013

Citation: Staats CC, Kmetzsch L Schrank A and Vainstein MH (2013) Fungal zinc metabolism and its connections to virulence. Front. Cell. Infect. Microbiol. 3:65. doi: 10.3389/fcimb. 2013.00065
This article was submitted to the journal Frontiers in Cellular and Infection Microbiology.

Copyright (0) 2013 Staats, Kmetzsch, Schrank and Vainstein. This is an openaccess article distributed under the terms of the Creative Commons Attribution License (CC BY). The use, distribution or reproduction in other forums is permitted, provided the original author(s) or licensor are credited and that the original publication in this journal is cited, in accordance with accepted academic practice. No use, distribution or reproduction is permitted which does not comply with these terms. 